\title{
Manual Removal versus Spontaneous Delivery of the Placenta at Cesarean Section: A Meta-Analysis of Randomized Controlled Trials
}

\author{
Meng-Chang Yang ${ }^{1} *$ \\ Peng $\mathrm{Li}^{1, *}$ \\ Wen-Jie Su' \\ Rong Jiang' \\ Jia Deng' \\ Ru-Rong Wang ${ }^{2}$ \\ Chao-Li Huang ${ }^{3}$
}

'Department of Anesthesiology, Sichuan Provincial People's Hospital, University of Electronic Science and Technology of China, Chengdu, People's Republic of China; ${ }^{2}$ Department of Anesthesiology, West China Hospital, Sichuan University, Chengdu, People's Republic of China; ${ }^{3}$ Department of Internal Medicine, Eastern Hospital, Sichuan Provincial People's Hospital, University of Electronic Science and Technology of China, Chengdu, People's Republic of China

*These authors contributed equally to this work

Correspondence: Ru-Rong Wang Department of Anesthesiology, West China Hospital, Sichuan University, Chengdu, Sichuan, People's Republic of China

Tel +86 I8I40049936

$\mathrm{Fax}+86$ 28-87393632

Email wangrurong@scu.edu.cn

Chao-Li Huang

Department of Internal Medicine, Eastern Hospital, Sichuan Provincial People's Hospital, University of Electronic Science and Technology of China, No. 585, Damian, Honghe North Road, Longquanyi District, Chengdu, People's Republic of China

Tel +86 I335085568|

Fax +86 28-8777568।

Email chaoli_huang@126.com
Purpose: Several randomized clinical trials (RCTs) investigated the effects of the manual placental removal on hemorrhage or other hemorrhage-related complications compared with the spontaneous placental removal during cesarean section (CS), while the results remained controversial and were inconsistent. The purpose of this meta-analysis was to quantify the pooled effects of the methods of placental removal on hemorrhage during CS.

Patients and Methods: A systematic literature search was conducted using PubMed, EMBASE, Web of Science, and Google Scholar. Heterogeneity was tested by $I^{2}$ statistics and Q-statistic. The random-effects model or fixed-effects model were used to calculate the pooled effect for the included studies according to heterogeneity. And the term of standardized mean difference (SMD) with $95 \%$ confidence intervals (CI) was pooled and estimated the effects across all studies.

Results: A total of nine RCTs were included in this meta-analysis. Compared with spontaneous group, manual placental removal increased the amount of hemorrhage $(S M D=0.53$, $95 \%$ CI $[0.12,0.94] ; Z=2.54, P=0.011)$ and increased the risk of endometritis $(O R=1.84$, $95 \%$ CI $[1.31,2.58] ; Z=3.52, P<0.0001)$. In contrast, there was no significant difference concerning the operating time $(S M D=-0.30,95 \% \mathrm{CI}[-0.85,0.24] ; Z=1.09, P=0.276)$, the length of hospital stays $(S M D=0.11,95 \% \mathrm{CI}[-0.08,0.30] ; Z=1.11, P=0.265)$, and blood transfusion requirement $(O R=1.36,95 \%$ CI [0.91, 2.04]; $\mathrm{Z}=1.52, P=0.129)$, respectively. Conclusion: Comparing with spontaneous placental removal, manual placental removal appeared to be less positive effect during CS. Because of the limitations of this metaanalysis, more high-quality RCTs are needed to confirm our findings.

Keywords: cesarean section, manual placental removal, spontaneous placenta removal, hemorrhage, meta-analysis

\section{Introduction}

Cesarean section (CS) is a life-saving surgery when certain complications occur during pregnancy and childbirth. ${ }^{1} \mathrm{CS}$ rates have consistently increased in both developing and developed countries in the recent few decades. ${ }^{2}$ Based on the recent data from 150 countries, currently, CS accounts for $18.6 \%$ of all births, and for the least and most developed regions ranged from $6 \%$ to $27.2 \%$, respectively. ${ }^{1}$ Despite advances in modern surgical equipment and postoperative care, related risks such as hemorrhage, iatrogenic tumors, thromboembolic events, and infection are still potential threats. ${ }^{3}$ Patients with malignant placenta may have influence on the blood loss and clinical outcomes of cesarean section. Additional therapeutic 
methods can reduce blood loss during cesarean delivery and preserve fertility in pernicious placenta previa patients who are complicated with placenta accrete. ${ }^{4}$ Among them, hemorrhage is one of the most common complications of delivery and is considered to be the main cause of preventable maternal mortality in the world. ${ }^{5}$ Meanwhile, compared with vaginal delivery, cesarean delivery has a higher risk of hemorrhage. ${ }^{6}$ Estimating the amount of blood loss during CS is critical to reducing surgically induced morbidity. ${ }^{7}$ However, due to its extremely hard accuracy and poorly reproducible, it is usually underestimated. ${ }^{8}$ As complications of CS may cause lifethreatening bleeding, appropriate procedures should be taken to reduce intraoperative and postoperative blood loss. ${ }^{9}$ The type of uterine incision and the method of placental removing are important factors in determining the outcomes during CS, such as the amount of blood loss. Compared with lower vertical incision or classic incision, lower transverse uterine incision has less operative blood loss. Patients with lower vertical and classic incision could increase operative blood loss. ${ }^{10-12}$

The method of placental removing is one such procedure that can affect outcomes of cesarean delivery, such as the amount of bleeding during intraoperative and postoperative, the time of operation, the occurrence of postoperative endometritis ${ }^{13,14}$ and may contribute to an increase or decrease in the incidence of $\mathrm{CS}^{3}{ }^{3}$ But the ideal method of placental removal during CS is still a controversial issue. ${ }^{15}$ The choice mainly bases on the surgeon's preference. At present, the research of placenta resection technology mainly focus on "manual" or "spontaneous" removal. ${ }^{14,16}$ Manual placental removal remains a conflicting issue owing to the risk of postpartum hemorrhage, postpartum endometritis, and placental abnormalities in subsequent pregnancies. ${ }^{13,15,17}$

Previously, several longitudinal studies and randomized clinical trials have suggested that manual removal of the placenta increased risks of postpartum blood loss, postpartum endometritis, and placental abnormalities. A longitudinal linked national cohort study by Ruiter et al concluded that the incidence of hemorrhage was higher in women with manual placental removal compared to women have no history of manual placental removal. ${ }^{18}$ A randomized clinical trial by Baksu et al suggested that manual placental removal during CS could lead to more blood loss and a higher risk of postpartum endometritis, compared with method of spontaneous. ${ }^{19}$ Dabashi et al reported that manual placental delivery increased the risk of blood loss and endometritis compared with the spontaneous way of placental removal. ${ }^{20}$ Similarly, Hider et al observed that compared with the way of spontaneous, manual removal of the placenta during CS significantly increased perioperative hemorrhage and maternal infection rates. ${ }^{13}$ However, some other studies showed that it had no significant effect on blood loss. A randomized clinical trial by Gun et al concluded that there was no correlation between the way of removal of the placenta and hemorrhage in CS deliveries. ${ }^{3}$ Chandra et al also showed that both postoperative endometritis and bleeding were independent of the method of placental delivery. ${ }^{21}$ Gol et al found that compared with natural separation, manual placental removal without a significant relationship with increased bleeding; Also, in terms of the incidence of postoperative complications or postoperative hemoglobin levels, manual placental removal without influence. ${ }^{22}$ A prospective multicenter trial reported that manual removal of the placenta and intrauterine cleaning have no adverse effects on maternal blood loss and infectious morbidity after elective cesarean section. ${ }^{23}$

All the studies above demonstrated that results from the randomized clinical trials or other type studies did not indicate a consistent conclusion. Whether manual removal of the placenta increases blood loss or other complications related to blood loss during CS remained a controversial issue. In the present study, we carried out a comprehensive search and meta-analysis to investigate the effect of manual removal of the placenta on blood loss and other complications compared with the spontaneous placenta removal during $\mathrm{CS}$.

\section{Materials and Methods}

We developed this meta-analysis according to the Preferred Reporting Item for Systematic Review and Meta-Analysis Protocols statement. ${ }^{24}$

\section{Literature Search Strategy}

A comprehensive and systemic retrieval was conducted in various databases, containing PubMed, EMBASE, and Web of Science We searched all published RCTs of manual and spontaneous placenta removal in CS. The latest search was updated on July 1, 2020. We used a combination of keywords for retrieval ("Manual 
removal of placenta OR manual placental removal OR manual placental separation" and "spontaneous placental delivery OR spontaneous placental separation" OR "removal of the placenta OR placental separation OR placental removal" and "cesarean section OR cesarean delivery" and "hemorrhage OR bleeding OR blood loss" AND "Randomized Controlled Trial OR RCT"). To further find potentially relevant studies, we manually scanned the references of all selected articles, and read recent reviews. Two authors carefully reviewed each identified report.

\section{Inclusion Criteria and Study Selection}

Studies were included if they satisfied the following inclusion criteria: 1) the mode of delivery was cesarean section; 2) randomized clinical trials; 3 ) reporting at least one measurement and other complications related to blood loss; 4) two groups were treated with the manual placenta and spontaneous separation, respectively.

Studies were excluded if they met the following exclusion criteria: 1) the study involved only one form of placental separation; 2) the methods of placenta separation were a mixture of manual placenta and spontaneous separation and other factors; 3) studies have no full-text and conference abstracts; 4) case reports, cohort studies, and animal trails; 5) not written in English. Two authors separately selected titles and abstracts and subsequently full-text articles. Discuss the disagreement with the third author and adjust the inconsistent after reaching a consensus.

\section{Data Extraction and Study Quality Assessment}

Two authors carefully and independently read the full text of retrieved included articles. The extracted information of eligibility studies contained publication year, first author's name, sample size, blood loss in the spontaneous dissection group and manual group, the operating time, the length of postoperative hospital stay, postoperative complications (endometritis and), and conclusion. In ordered to assess the quality of the articles included in this analysis, we applied the modified Jadad scale ranging from 0 (minimum) to 8 (maximum) points. It contained eight quality criteria. The higher the score of the study, the better its quality.

\section{Statistical Analysis}

The data analysis was performed using stata 14.0 software. The pooled measure of the effect across the included studies was estimated by the term of standardized mean difference (SMD) and 95\% confidence intervals (CI). When there were categorical variables, odds ratios (OR) were calculated for each study. The level of heterogeneity was explored using the $I^{2}$ statistic and Q-statistic. The $I^{2}$ index expresses the proportion of true heterogeneity in the observed variance. An $I^{2}$ value of $0 \%$ indicates that no heterogeneity is observed, and a larger value indicates an increase in heterogeneity. Therefore, percentages of about $25 \%\left(I^{2}=25\right), 50 \%\left(I^{2}=50 \%\right)$, and $75 \%\left(I^{2}=75 \%\right)$ would explain low, medium, and high heterogeneity, respectively. The Q-statistic is a measure of the real variance among studies. A significant Q-statistic demonstrates heterogeneity within studies. If there was no heterogeneity $(P \geq 0.05$, $I^{2}<50 \%$ ), a fixed-effect meta-analysis was used, otherwise $(P<0.05)$ a random-effects meta-analysis was used. SMD, $95 \% \mathrm{CI}$, and the pooled effect sizes were expressed by forest plots. The Z-test examined the significance of the pooled effect. A $P$ value below 0.05 was considered statistically significant.

\section{Results}

Figure 1 displays the flow chart of the study systematic retrieve. The initial literature search discovered 1296 articles, including 894 duplicates which were deleted at first. Based on titles and abstracts 389 articles clearly did not meet our inclusion criteria and were eliminated then. For the remaining thirteen studies, the full texts were screened, and four studies did not satisfy our inclusion criteria. Finally, nine randomized controlled trials were included in this meta-analysis.

The baseline characteristics of the included articles are shown in Table 1. A total of nine RCTs containing 1248 cases of the manual group and 1252 cases of the spontaneous group were included in this meta-analysis. Five studies reported that all patients undergo transverse lower segment CS, one studies undergo low uterine vertical incisions, three studies did not mention the type of incision. Quality assessment results based on eight quality criteria as shown in Table 2. The Jadad scores of the five studies were above 5 , with high quality. 


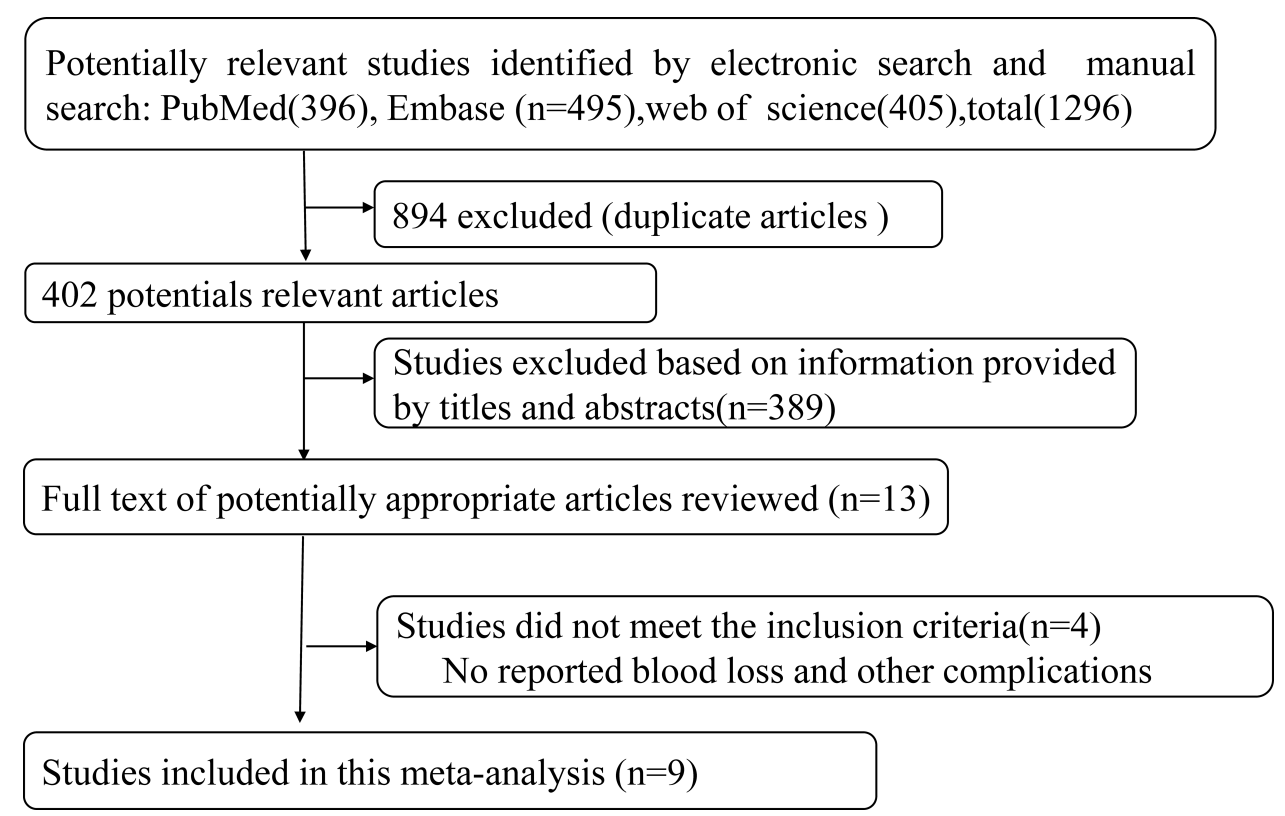

Figure I Flowchart of search and study selection.

\section{Hemorrhage}

Six studies reported the difference in blood loss between the manual group and spontaneous group. The heterogeneity test showed significant heterogeneity across these studies $\left(I^{2}=\right.$ 93.9\%, $P<0.001$; Figure 2), therefore the random-effects model was used. The pooled results indicated that statistical difference was found between the manual group and spontaneous group in terms of hemorrhage $(S M D=0.53,95 \% \mathrm{CI}$ $[0.12,0.94] ; Z=2.54, P=0.011$; Figure 2). The amount of bleeding with manual placental removal was more than with spontaneous placental delivery.

\section{The Operating Time}

A comparison of the operating time between the manual group and spontaneous group was obtainable in six studies. This random-effects meta-analysis showed that there was no statistical difference in the operating time between the two methods of placental removal ( $S M D=-0.30,95 \%$ CI [-0.85, 0.24]; $Z=1.09, P=0.276$ ), with high evidence of the heterogeneity $\left(I^{2}=96.0 \%, P<0.001\right.$; Figure 3$)$.

\section{The Length of Postoperative Hospital Stays}

Five articles reported the comparison of the length of postoperative hospital stays between the manual group and spontaneous group. The pooled results revealed that there was no significant difference in the length of postoperative hospital stay between the manual group and spontaneous group $(S M D=0.11,95 \%$ CI $[-0.08,0.30]$; $Z=1.11, P=0.265)$, with medium evidence of the heterogeneity $\left(I^{2}=74.4 \%, P<0.001\right.$; Figure 4$)$.

\section{Postoperative Complications}

Seven articles reported the occurrence of endometritis and blood transfusion requirements after the cesarean section. Pooled effects by a fixed-effects model demonstrated there was a difference in the incidence of endometritis between cesarean section placental manual removal group and spontaneous removal group $(O R=1.84,95 \%$ CI [1.31, 2.58]; $Z=3.52, P<0.0001$ ); in terms of blood transfusion requirement, there was no difference in the blood transfusion requirement between cesarean section placental manual removal group and spontaneous removal group $(O R=1.36,95 \%$ CI $[0.91,2.04] ; \mathrm{Z}=1.52, P=0.129)$, without significant heterogeneity $\left(I^{2}=0.0 \%, P=0.531\right.$; Figure 5). The incidence of endometritis was higher in the manual removal group than in the spontaneous removal group.

\section{Discussion}

In the current published studies, the mode of placental removal is still a conflicting issue because of the risk of 


\begin{tabular}{|c|c|c|c|c|c|c|c|c|c|c|c|c|c|c|c|c|c|c|c|}
\hline \multicolumn{2}{|c|}{$\begin{array}{l}\frac{n}{0} \\
\frac{.0}{\underline{n}} \\
\frac{\underline{s}}{y}\end{array}$} & \multicolumn{2}{|l|}{ 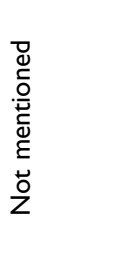 } & \multicolumn{2}{|c|}{ 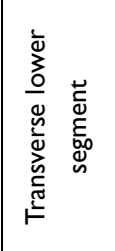 } & \multicolumn{2}{|c|}{ 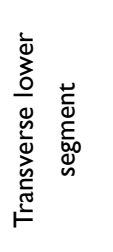 } & \multicolumn{2}{|c|}{ 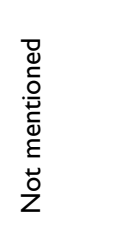 } & \multicolumn{2}{|c|}{ 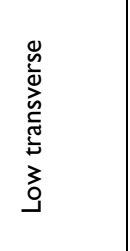 } & \multicolumn{2}{|c|}{ 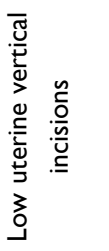 } & \multicolumn{2}{|l|}{ 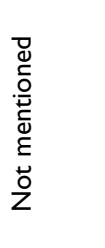 } & \multicolumn{2}{|c|}{ 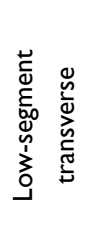 } & \multicolumn{2}{|c|}{ 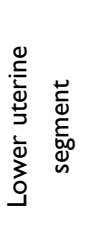 } \\
\hline \multicolumn{2}{|c|}{ 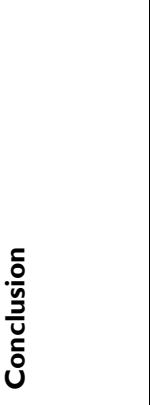 } & \multicolumn{2}{|l|}{ 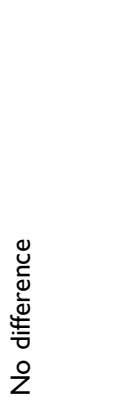 } & \multicolumn{2}{|c|}{ 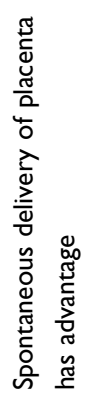 } & \multicolumn{2}{|l|}{ 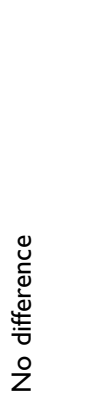 } & \multicolumn{2}{|c|}{ 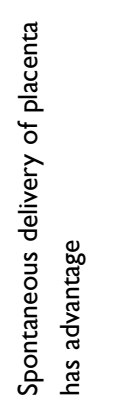 } & \multicolumn{2}{|c|}{ 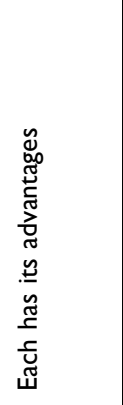 } & \multicolumn{2}{|l|}{ 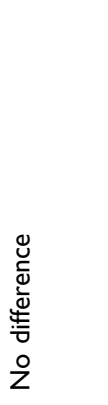 } & \multicolumn{2}{|c|}{ 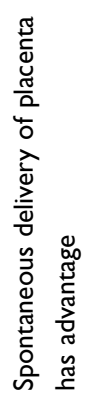 } & \multicolumn{2}{|c|}{ 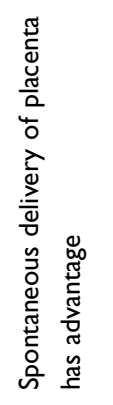 } & \multicolumn{2}{|c|}{ 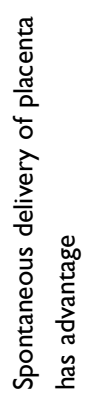 } \\
\hline 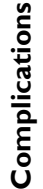 & 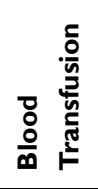 & ? & ? & $\stackrel{\infty}{+}$ & $\bar{m}$ & $\stackrel{\mathrm{O}}{\mathrm{z}}$ & O & O & $\stackrel{\mathrm{o}}{\mathrm{z}}$ & in & 0 & 0 & 0 & $m$ & $\theta$ & O & Q & Q & ? \\
\hline 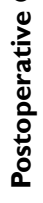 & 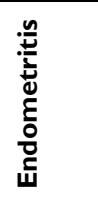 & $\underline{a}$ & $\underline{m}$ & $z$ & z & $\stackrel{\mathrm{O}}{\mathrm{z}}$ & $z$ & $\stackrel{\circ}{z}$ & o & $\sigma$ & $\sigma$ & in & $\sigma$ & O & O & $\stackrel{\infty}{\infty}$ & $q$ & N & - \\
\hline
\end{tabular}

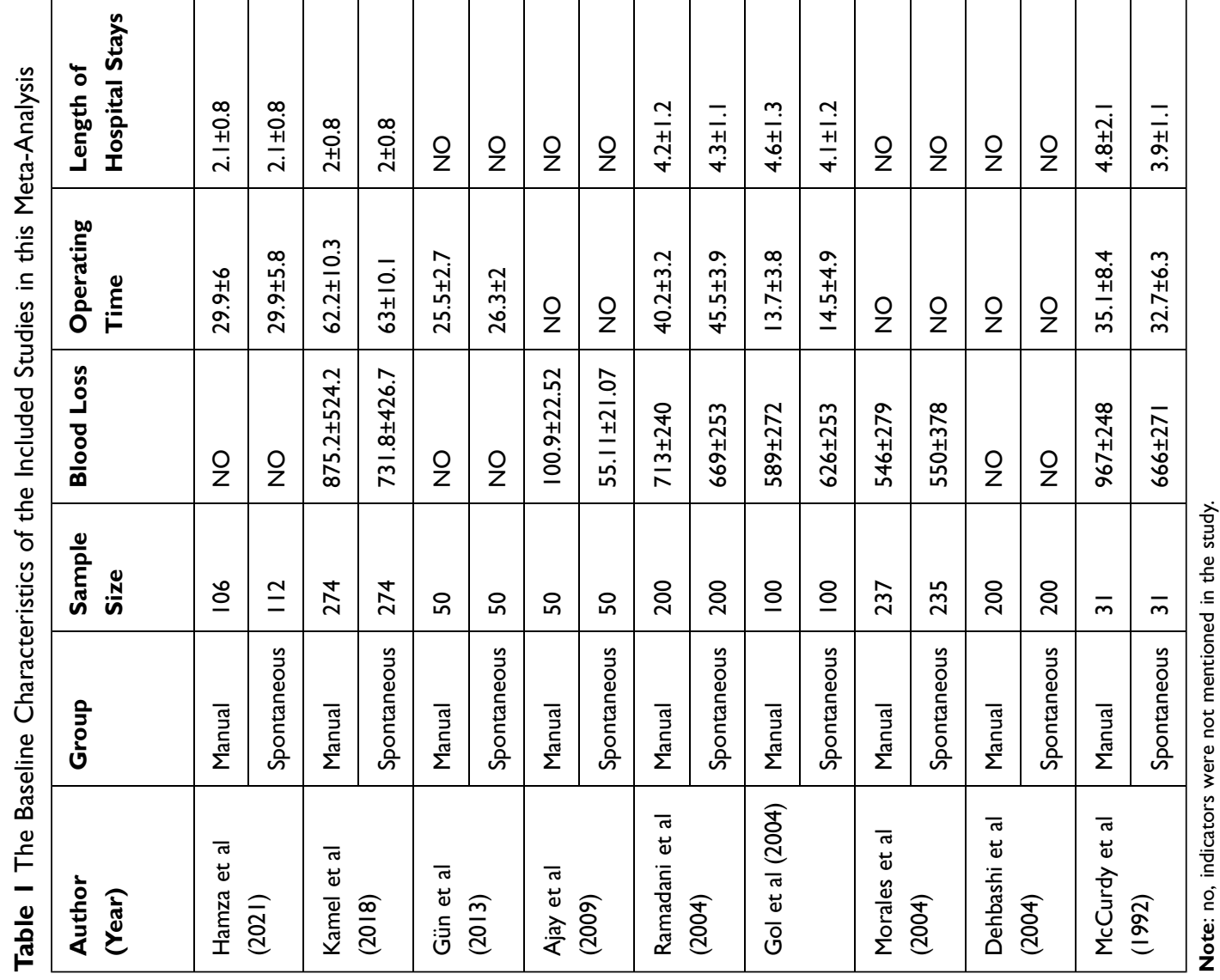




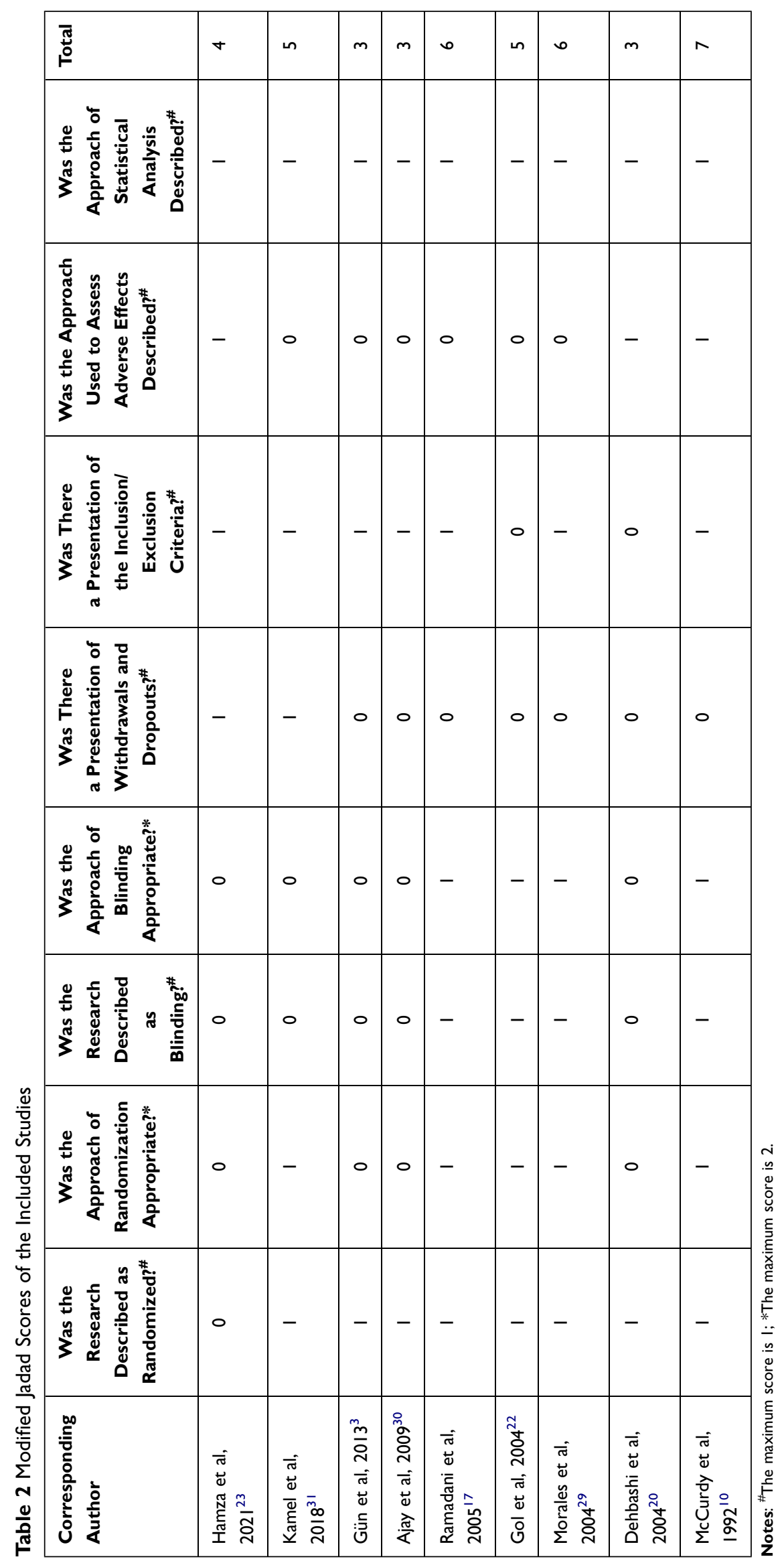




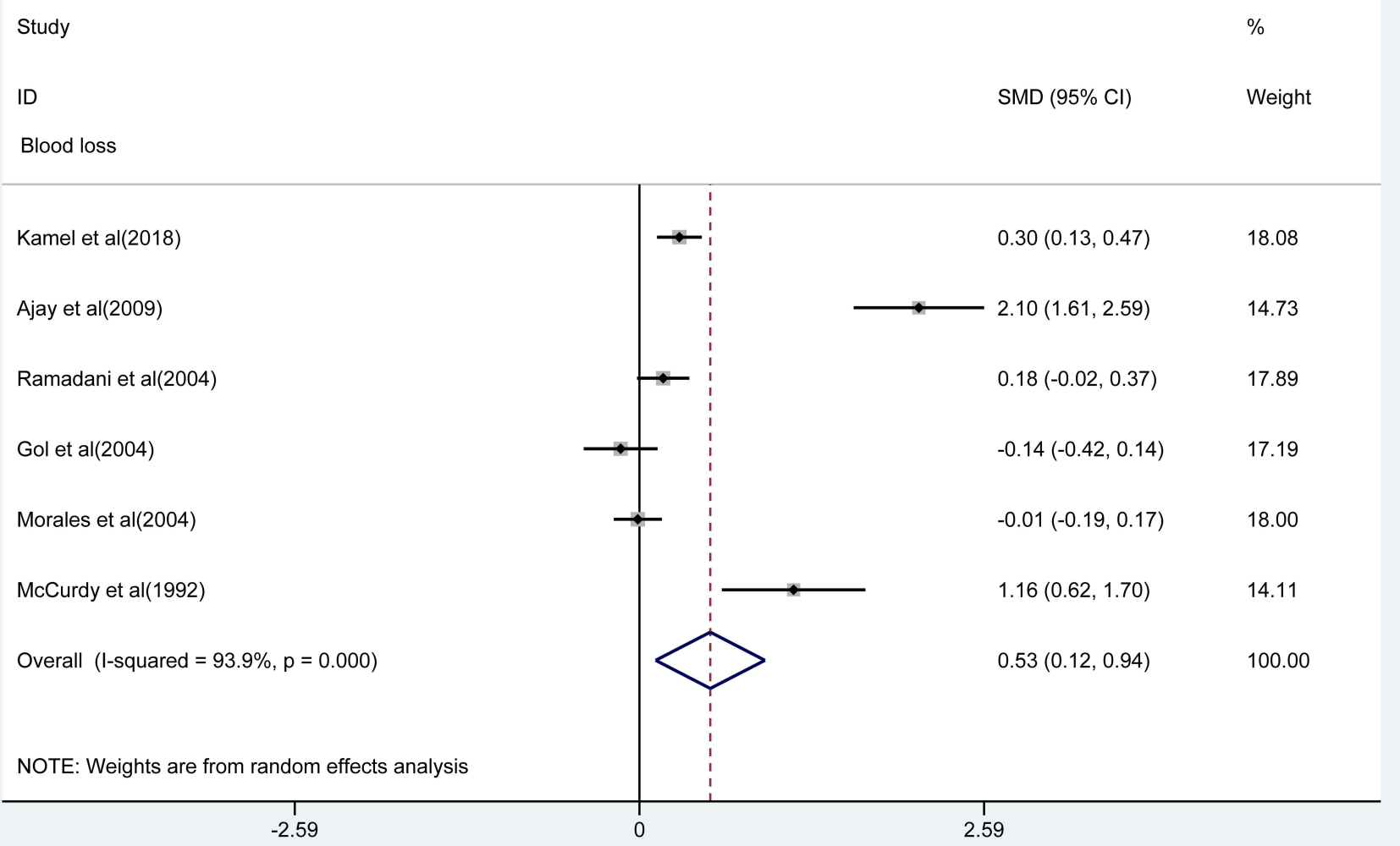

Figure 2 Forest plot of hemorrhage when using manual and spontaneous.

hemorrhage, and other complications related to hemorrhage. This meta-analysis explored whether the manual placental removal during CS affects hemorrhage and other complications compared with spontaneous placental removal. Nine RCTs published were included in this analysis. The results of this study demonstrated that the risks of hemorrhage and endometritis in CS were increased by the manual method of placental removal compared with the spontaneous placental delivery. In contrast, this way failed to reduce operative time, the length of hospital stays, and the blood transfusion requirement. Manual method of placental removal may put women at undue risk with no added benefit.

We found significant hemorrhage in the manual method of placental removal group. This was consistent with other authors' findings. ${ }^{10,12}$ The current results were comparable with a published review of Anorlu et al, who reported that Manual placenta removal was associated with more hemorrhage (weighted mean difference
[WMD] 94.42, 95\% CI $[17.19, \quad 171.64]) .^{25}$ From a mechanistic view, after the fetal is removed, the uterine muscles begin to contraction and retraction immediately, thereby lessening the size of the uterus; As the uterus becomes smaller, the size of the placental bed is significantly smaller than that of the incompressible placenta; This causes shear movement, which results in separation of the placenta and compression of the new exfoliated vessels supplying the placental bed, thereby reducing blood loss; This mechanism may explain why spontaneous placental separation causes less blood loss than manual placental dissection. ${ }^{25}$ Manual placental dissection could cause the problem of fetal membrane residue and affect the contractile function of uterus, which is an important factor in the increase of bleeding. Abnormal placental attachment, placental adhesion or retention are the most common placental factors of bleeding during manual separation of the placenta. In contrast, some authors found no difference between either method. ${ }^{11,21,22}$ Gol 
Study

ID

Operating time

Hamza et al(2021)

Kamel et al (2018)

Gün et al(2013)

Ramadani et al(2004)

Gol et al(2004)

McCurdy et al(1992)

Overall (I-squared $=96.0 \%, p=0.000)$

NOTE: Weights are from random effects analysis
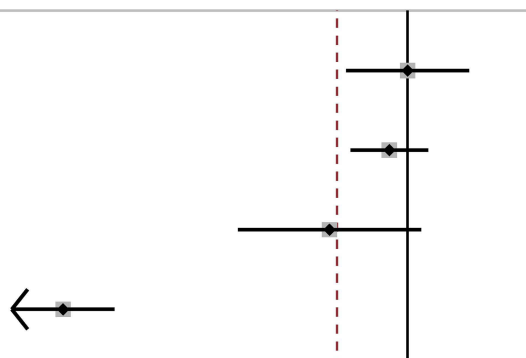

$0.00(-0.27,0.27)$

16.96

$-0.08(-0.25,0.09) \quad 17.38$

$-0.34(-0.73,0.06) \quad 16.18$

$-1.49(-1.71,-1.26) \quad 17.17$

$-0.18(-0.46,0.10) \quad 16.90$

$0.32(-0.18,0.82) \quad 15.39$

$-0.30(-0.85,0.24) \quad 100.00$

Figure 3 Forest plot of operating time when using manual and spontaneous.

et $\mathrm{al}^{22}$ reported that manual removal of the placenta was not connected with any significant risk of bleeding, this may be due to the clamping of the incision and the use of oxytocin, which is the most crucial factors in preventing excessive hemorrhage during CS. An additional important factor that affects the amount of bleeding during CS was the type of uterine incision. ${ }^{22}$ Patients with vertical lower segment incision or classical upper segment incision are known for more blood loss than the transverse lower segment incision. ${ }^{17,19,22}$

In regard to endometritis, endometritis is the most common complication of cesarean section, with an incidence of between $5 \%$ and $85 \%$, depending on the patient population investigated. $^{26}$ In our study, pooled results showed that the risks of endometritis in CS was increased by the manual method of placental removal compared with the spontaneous placental delivery. Meanwhile, some studies have claimed that higher risks of endometritis. ${ }^{10,27}$ In the study of Anorlu et al, following manual placental removal, an increase in the risk of endometritis has been shown. ${ }^{25}$ It was speculated that manual placental removal will damage the local host's defense ability, and may bring bacteria into the endometrial cavity, leading to endometritis. However, in the study of Gol et al, the incidence of endometritis was no significant difference. $^{22}$ The study of Chandra et al studies found no difference in postoperative endometritis. ${ }^{21}$ Even in the spontaneous placental group, entering of foreign microbes (for example, through curettage or gauze used by a surgeon to remove clots and placental debris in the uterine cavity) can lead to endometritis. In theory, any foreign body entering the uterine cavity will cause a large number of microorganisms to enter the uterine cavity, even if the foreign body is a sterilized surgical glove. ${ }^{20}$ Therefore, if the patient's condition is stable, we think that it is better to allow the placenta to deliver naturally, which is consistent with Atkinson's finding that the risk of endometritis after artificial placenta extraction in cesarean section is significantly higher than that after assisted spontaneous placenta delivery. ${ }^{28}$ 
Study

ID

Length of hospital stays

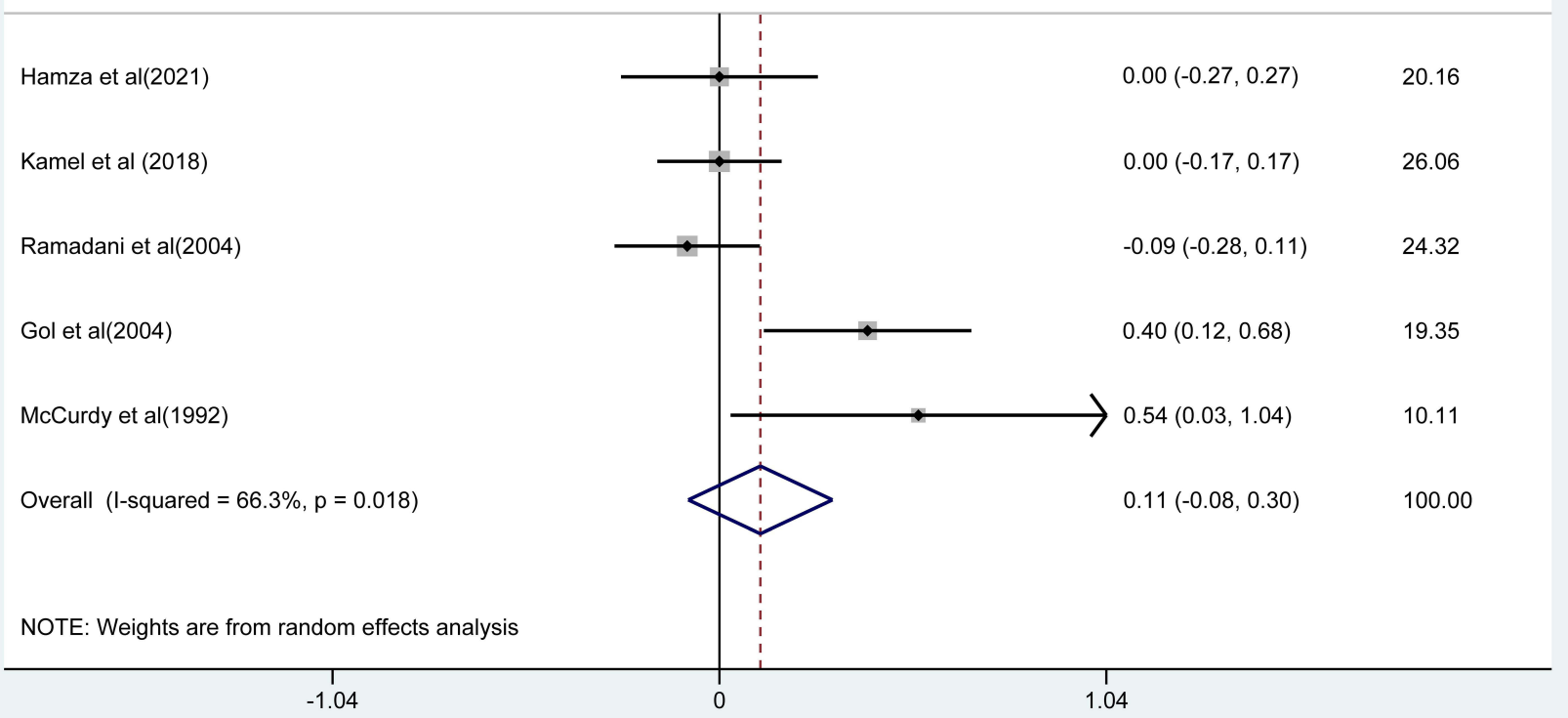

Figure 4 Forest plot of hospital stays when using manual and spontaneous.

In terms of the operating time, hospital stays, and the blood transfusion, without significant difference between two groups. McCurdy et al reported that the duration of the operating time, duration of operative stage III were not decreased by the methods of placental delivery. ${ }^{10}$ Ajay et al showed that the duration of surgery was not changed by the methods of placental separation. ${ }^{30}$ The study found no significant difference in blood transfusions between the two methods (there are very few of such studies).

There were some limitations in this meta-analysis. First, the limited number of studies satisfied our searching criteria, not all related randomized trials were included mainly because of publication bias or selection bias, and this might affect the robust of the results. Also, the considerable heterogeneity existed in the included studies, because of the differences in the type of incision, publication year and study quality. Subgroup analysis of different incision types cannot be carried out due to the limitation of the number of included studies. In our future study, the effect of incision type on bleeding should be considered. Second, even if we have used the random effect model, the combination of results may not be suitable for all outcome measurements. Finally, we did not assess the publication bias by funnel plot of the included studies, because each of the analysis indicators corresponds to a smaller than six included studies.

\section{Conclusion}

In summary, this meta-analysis demonstrated that manual placental removal has testified to increase blood loss during CS, with an increased incidence of endometritis. Manual placental removal failed to diminish the operative time, the length of hospital stays, and the blood transfusion requirement. As a result, such obstetric surgery puts women at inappropriate risk without additional benefit. The placenta can be spontaneous placental delivery after the maternal delivery of the fetus during cesarean section. If the amount of bleeding increases and there is no indication of spontaneous delivery of the placenta, manual placental removal can be considered. To effectively compare the two methods, further studies are recommended with a more standardized estimation of 
Study

ID

postoperative complications

\section{Endometritis}

Hamza et al(2021)

Ramadani et al(2004)

Gol et al(2004)

Dehbashi et al(2004)

McCurdy et al(1992)

Subtotal $(\mathrm{I}$-squared $=4.8 \%, p=0.380$ )

\section{Blood transfusion}

Kamel et al (2018)

Ramadani et al(2004)

Gol et al(2004)

Morales et al(2004)

Subtotal $(\mathrm{I}$-squared $=0.0 \%, p=0.531$ )

Overall (I-squared $=0.0 \%, p=0.489)$

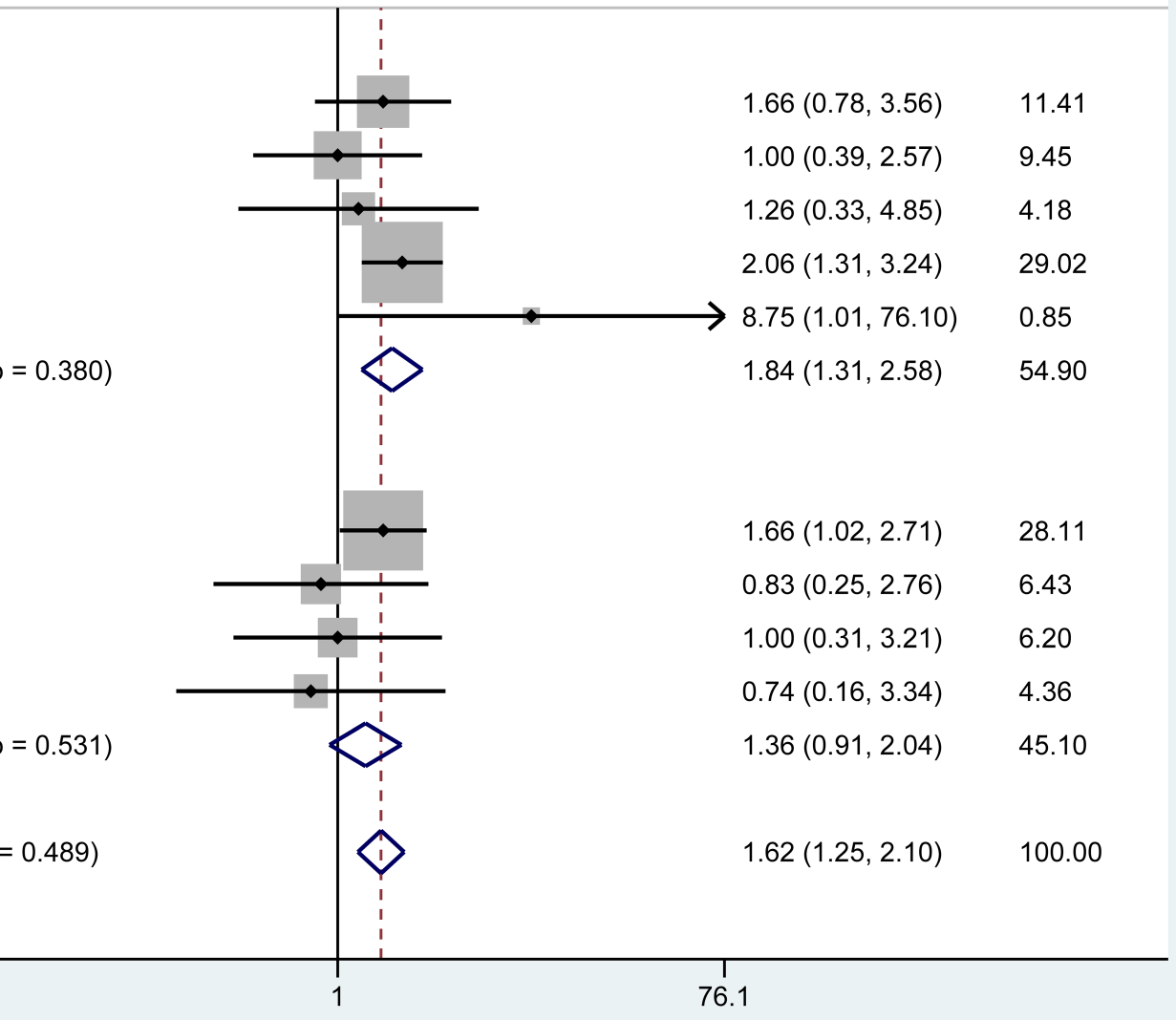

Figure 5 Forest plot of endometritis and blood transfusion when using manual and spontaneous.

blood loss. And large-scale randomized clinical trials are needed to further confirm our findings.

\section{Compliance with Ethical Standards}

This article does not contain any studies with human participants or animals performed by any of the authors.

\section{Acknowledgments}

Meng-Chang Yang and Peng Li are co-first authors for this study.

\section{Author Contributions}

All authors made substantial contributions to conception and design, acquisition of data, or analysis and interpretation of data; took part in drafting the article or revising it critically for important intellectual content; agreed to submit to the current journal; gave final approval of the version to be published; and agreed to be accountable for all aspects of the work.
$\%$

\section{OR $(95 \% \mathrm{Cl}) \quad$ Weight}


4. Wei Y, Luo J, Luo D. Comparison of efficacy between internal iliac artery and abdominal aorta balloon occlusions in pernicious placenta previa patients with placenta accrete. Gynecol Obstet Invest. 2019;84 (4):343-349. doi:10.1159/000494493

5. Van Lerberghe W, Manuel A, Matthews Z, Cathy W. The World Health Report 2005 - Make Every Mother and Child Count. World Health Organization; 2005.

6. Bateman BT, Berman MF, Riley LE, Leffert LR. The epidemiology of postpartum hemorrhage in a large, nationwide sample of deliveries. Anesth Analg. 2010;110(5):1368-1373. doi:10.1213/ ANE.0b013e3181d74898

7. Vimala N, Mittal S, Kumar S. Sublingual misoprostol versus oxytocin infusion to reduce blood loss at cesarean section. Int $J$ Gynecol Obstet. 2006;92(2):0-110. doi:10.1016/j.ijgo.2005.10.008

8. Brecher ME, Monk TG, Goodnough LT. A standardized method for calculating blood loss. Transfusion. 2010;37(10):1070-1074. doi:10.1046/j.1537-2995.1997.371098016448.x

9. Sekhavat L, Firouzabadi RD, Mojiri P. Effect of expansion technique of uterine incision on maternal blood loss in cesarean section. Arch Gynecol Obstet. 2010;282(5):475-479. doi:10.1007/s00404-0091251-5

10. Mccurdy CM, Magann EF, Mccurdy CJ, Saltzman AK. The effect of placental management at cesarean delivery on operative blood loss. Am J Obstet Gynecol. 1992;167(5):1363-1367. doi:10.1016/S00029378(11)91717-7

11. Magann EF, Washburne JF, Harris RL, Bass JD, Morrison JC. Infectious morbidity, operative blood loss, and length of the operative procedure after cesarean delivery by method of placental removal and site of uterine repair. J Am Coll Surg. 1995;181(6):517-520.

12. Magann EF, Dodson MK, Allbert JR, McCurdy CM, Martin RW, Morrison JC. Blood loss at time of cesarean section by method of placental removal and exteriorization versus in situ repair of the uterine incision. Surg Gynecol Obstet. 1993;177(4):389-392.

13. Hidar S, Jennane TM, Bouguizane S, Lassoued L, Bibi M, Khaïri H. The effect of placental removal method at cesarean delivery on perioperative hemorrhage: a randomized clinical trial isrctn 49779257. Eur J Obstet Gynecol Reprod Biol. 2004;117 (2):179-182. doi:10.1016/j.ejogrb.2004.03.014

14. Merchavy S, Levy A, Holcberg G, Freedman EN, Sheiner E. Method of placental removal during cesarean delivery and postpartum complications. Int $J$ Gynaecol Obstet. 2007;98(3):0-236. doi:10.1016/j.ijgo.2007.04.002

15. Ahmed A, Mohamed E, Hytham A, Ibrahim A, Mohamed K, Ahmed A. How can methods of placental delivery in cesarean section affect perioperative blood loss? A randomized controlled trial of controlled cord traction versus manual removal of placenta. J Obstet Gynaecol Res. 2018;45:133-140.

16. Kaya B, Guralp O, Daglar K, et al. Extra-abdominal removal of placenta during cesarean section: a prospective randomized controlled trial of a novel technique. $J$ Perinat Med. 2016;44. doi:10.1515/jpm-2015-0330

17. Ramadani H. Cesarean section intraoperative blood loss and mode of placental separation. Digest World Latest Med Infor. 2005;87 (2):114-118.
18. Ruiter L, Kazemier BM, Mol BWJ, Pajkrt E. Incidence and recurrence rate of postpartum hemorrhage and manual removal of the placenta: a longitudinal linked national cohort study in the Netherlands. Eur J Obstet Gynecol Reprod Biol. 2019;238:114-119. doi:10.1016/j.ejogrb.2019.05.022

19. Baksu A, Kalan A, Ozkan A, Baksu B, Tekelioğlu M, Goker N. The effect of placental removal method and site of uterine repair on postcesarean endometritis and operative blood loss. Acta Obstet Gynecol Scand. 2005;84(3):266-269. doi:10.1111/j.0001-6349.200 5.00729.x

20. Dehbashi S, Honarvar M, Fardi FH. Manual removal or spontaneous placental delivery and postcesarean endometritis and bleeding. Int J Gynecol Obstet. 2004;86(1):0-15.

21. Chandra P, Schiavello HJ, Kluge JE, Holloway SL. Manual removal of the placenta and postcesarean endometritis. $J$ Reprod Med. 2002;47(2):101-106.

22. Gol M, Baloglu A, Aydin C, Ova L, Yensel U, Karci L. Does manual removal of the placenta affect operative blood loss during cesarean section? Eur J Obstet Gynecol Reprod Biol. 2004;112(1):57-60. doi:10.1016/s0301-2115(03)00278-1

23. Hamza H, Rezk M, Tharwat A, Amgad M, Dawood R. Impact of manual removal of the placenta and intrauterine cleaning during elective cesarean delivery on maternal infectious morbidity and blood loss. J Matern Fetal Neonatal Med. 2021;1-5. doi:10.1080/ 14767058.2021.1875442

24. Shamseer L, Moher D, Clarke M, et al. Preferred reporting items for systematic review and meta-analysis protocols (prisma-p) 2015 statement. BMJ. 2015;349(1):g7647-g7647. doi:10.1136/bmj.g7647

25. Methods of delivering the placenta at caesarean section. Obstet Gynecol. 2008;112(5):1173-1174. doi:10.1097/01.AOG.0000336 $080.35574 .6 \mathrm{~d}$

26. Yancey MK, Clark P, Duff P. The frequency of glove contamination during cesarean delivery. Obstet Gynecol. 1994;83(4):538. doi:10.1097/00006250-199404000-00009

27. Lasley DS, Eblen A, Yancey MK, Duff P. The effect of placental removal method on the incidence of postcesarean infections. Am $J$ Obstet Gynecol. 1997;176(6):1250-1254. doi:10.1016/S00029378(97)70342-9

28. Atkinson MW, Owen J, Wren A, Hauth JC. The effect of manual removal of the placenta on post-cesarean endometritis. Obstet Gynecol. 1996;87(1):99-102. doi:10.1016/0029-7844(95)00359-2

29. Morales M, Ceysens G, Jastrow N, et al. Spontaneous delivery or manual removal of the placenta during caesarean section: a randomised controlled trial. BJOG. 2004;111(9):908-912.

30. Ajay G, Suman A. Spontaneous delivery or manual removal of the placenta during cesarean section: A randomized controlled trial. $J$ Obstet Gynecol India. 2009;59(2):127-130.

31. Kamel A, El-Mazny A, Salah E, Ramadan W, Hussein AM, Hany A. Manual removal versus spontaneous delivery of the placenta at cesarean section in developing countries: a randomized controlled trial and review of literature. J Matern Fetal Neonatal Med. 2018;31 (24):3308-3308. doi:10.1080/14767058.2017.1369522
Therapeutics and Clinical Risk Management

\section{Publish your work in this journal}

Therapeutics and Clinical Risk Management is an international, peerreviewed journal of clinical therapeutics and risk management, focusing on concise rapid reporting of clinical studies in all therapeutic areas, outcomes, safety, and programs for the effective, safe, and sustained use of medicines. This journal is indexed on PubMed Central, CAS,
EMBase, Scopus and the Elsevier Bibliographic databases. The manuscript management system is completely online and includes a very quick and fair peer-review system, which is all easy to use. Visit http://www.dovepress.com/testimonials.php to read real quotes from published authors. 